\title{
Changes in HRCT findings in patients with respiratory bronchiolitis-associated interstitial lung disease after smoking
} cessation

\author{
M. Nakanishi*, Y. Demura*, S. Mizuno*, S. Ameshima*, Y. Chiba\#, I. Miyamori*, \\ H. Itoh ${ }^{\top}$, M. Kitaichi ${ }^{+}$and T. Ishizaki ${ }^{\S}$
}

ABSTRACT: High-resolution computed tomography (HRCT) findings in patients with respiratory bronchiolitis-associated interstitial lung disease (RB-ILD) are varied and nonspecific. There is no known report of changes in HRCT findings and respiratory function test results for RB-ILD patients following the cessation of smoking.

Five patients with RB-ILD, confirmed by surgical lung biopsy, were retrospectively studied. Each stopped cigarette smoking and did not receive corticosteroid therapy after diagnosis. The clinical symptoms, respiratory function test results and HRCT findings obtained at the final observation were compared with those from the time of diagnosis.

Ground-glass opacity and centrilobular nodules corresponding to pathological respiratory bronchiolitis, as well as intralobular fine linear-reticular opacity corresponding to fibrosis involving the subpleural alveolar septa, showed computed tomography-pathological correlations. Both clinical symptoms and the diffusing capacity of the lungs for carbon monoxide improved significantly following smoking cessation, as did ground-glass opacity and centrilobular nodules seen during the initial HRCT examination.

Centrilobular nodules and ground-glass opacity, which are the main features of high-resolution computed tomography of respiratory bronchiolitis-associated interstitial lung disease patients and represent pathological respiratory bronchiolitis, can be improved by smoking cessation. The diffusing capacity of the lung for carbon monoxide in respiratory function tests can be also improved.

KEYWORDS: High-resolution computed tomography, interstitial lung disease, respiratory bronchiolitis, respiratory bronchiolitis-associated interstitial lung disease, smoking cessation

$\mathbf{R}$ espiratory bronchiolitis (RB), first described in 1974 [1], is found nearly exclusively in cigarette smokers. The most characteristic features of the disease are accumulations of tan-brown macrophages in the lumen of the respiratory bronchiole and adjacent alveoli, along with mild chronic inflammation in the wall of respiratory bronchioles, which are associated with slight fibrosis, smooth muscle hypertrophy and thickening of the adjacent alveolar walls [2]. $\mathrm{RB}$ is of little clinical significance in the vast majority of cases $[1,3,4]$; however, in a small number, symptomatic interstitial lung disease may occur in association with the condition, which has been termed respiratory bronchiolitis-associated interstitial lung disease (RB-ILD) [5, 6]. RBILD is now recognised as a clinical and histological entity that is different from other types of idiopathic interstitial pneumonia [7], although it is considered to be difficult to distinguish from desquamative interstitial pneumonia (DIP) both clinically and pathologically [8-13].

Several reports have indicated that RB-ILD is improved by smoking cessation and corticosteroid administration with or without immunosuppressive agents $[5,6,9,12,14-16]$, and patients with RB-ILD have shown subjective and objective improvements with smoking cessation only [5, 14]. However, there are no known studies that have assessed those improvements sequentially using high-resolution computed tomography (HRCT) findings and respiratory function test results after smoking cessation alone. Other
AFFILIATIONS

*Third Dept of Internal Medicine,

${ }^{\S}$ Nursing Science, and

"Radiology, Medical Faculty,

University of Fukui, and

${ }^{\#}$ Thoracic Surgery, National Hospital

Organisation, Fukui Hospital, Turuga,

Fukui, and

+Pathology, National Hospital Organisation, Kinki-Chuo Chest Medical Center, Sakai, Osaka, Japan.

CORRESPONDENCE

M. Nakanishi

Third Dept of Internal Medicine

Medical Faculty

University of Fukui

23 Shimoaizuki Matsuoka-cho

Fukui Prefecture

910-1193

Japan

Fax: 81776618111

E-mail: mnakanishi@nifty.ne.jp

Received:

February 012006

Accepted after revision:

November 152006

STATEMENT OF INTEREST

None declared. 
reported cases of RB-ILD showed an unchanged or deteriorated condition in spite of smoking cessation and corticosteroid therapy $[9,12]$. Furthermore, HRCT findings in patients with RB-ILD, which include mainly centrilobular nodules and ground-glass attenuation, with occasional linear-reticular patterns and, rarely, honeycombing, are nonspecific and varied $[9,13,15,17]$; the results of respiratory function tests are also variable in published reports $[9,12,15]$. In addition, little is known regarding what kind of HRCT and respiratory functional abnormalities are improved or remain unchanged by smoking cessation, either with or without corticosteroid therapy. It also remains unclear whether RB-ILD has components of interstitial lung diseases (ILD) other than RB.

In the present study, the changes in HRCT findings and pulmonary function test results in RB-ILD patients were investigated after smoking cessation without corticosteroid therapy. To clarify the significance of each abnormal HRCT finding in the five RB-ILD patients, the present authors also attempted to determine how pathological abnormalities were represented by improved or unchanged HRCT findings following smoking cessation by linking them to changes in pulmonary function test results.

\section{MATERIALS AND METHODS \\ Patients}

Among patients who underwent a surgical lung biopsy for a pathological diagnosis of ILD between 1999 and 2004 at the
National Hospital Organisation Fukui Hospital (Turuga, Fukui, Japan), five cases diagnosed with RB-ILD were examined (table 1). Each patient was a heavy smoker at the time of diagnosis. The present authors were unable to identify any clinical or serological evidence of underlying connective tissue disease, or significant family history in any of the patients. One patient (case 3) had an occupational history of stone drilling and shaping; however, the period of that employment was $\sim 5 \mathrm{yrs}$ and the patient had stopped working 17 yrs prior to the diagnosis. All patients suffered from an insidious onset of cough that continued for 20-60 months (mean \pm SD $30.8 \pm$ 16.8 months), while three had an insidious onset of dyspnoea on exertion and two had sputum. HRCT, respiratory function and bronchoalveolar lavage (BAL) examinations were performed within a few days before a surgical lung biopsy. Each patient showed a reduction in the diffusing capacity of the lung for carbon monoxide (DL,CO), whereas blood gas analysis results were within normal limits. All had BAL findings that showed an increase in the number of macrophages. Each of the five patients was diagnosed as having RB-ILD based on the presence of RB in biopsy specimens, along with clinical and radiographical evidence of ILD.

Following the diagnosis of RB-ILD, all patients stopped smoking, following the present authors' recommendations, and none received corticosteroid therapy. The follow-up periods ranged 15-62 months $(46.4 \pm 20.8$ months). The present study was conducted retrospectively.

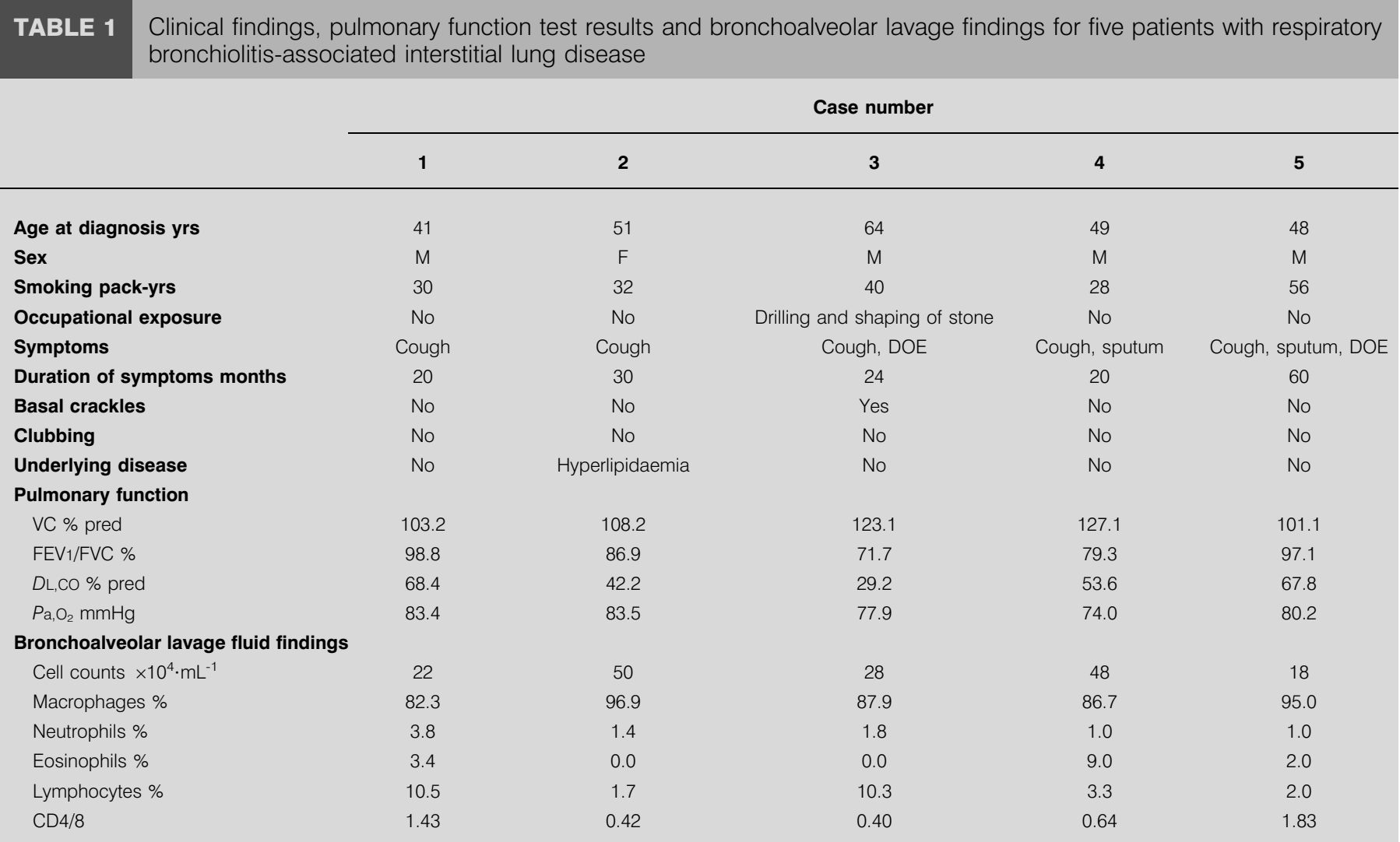

M: male; F: female; DOE: dyspnoea on exertion; VC: vital capacity; \% pred: \% predicted; FEV1: forced expiratory volume in one second; FVC: forced vital capacity; DL,CO: diffusing capacity of the lung for carbon monoxide; $\mathrm{Pa}, \mathrm{O}_{2}$ : arterial oxygen tension. $1 \mathrm{mmHg}=0.133 \mathrm{kPa}$. 


\section{Methods}

The initial HRCT findings and computed tomographypathological correlations were investigated based on the records of five patients diagnosed with RB-ILD. Clinical symptoms, respiratory function test results and HRCT findings that were obtained at the initial observation, during the followup period and at the final observation were compared. The outcome of each clinical course was assessed using the criteria of the International Consensus Statement of the American Thoracic Society/European Respiratory Society [18].

\section{Scoring of HRCT features}

HRCT abnormalities were scored using visual and semiquantitative methods, as reported previously $[19,20]$. The right and left lungs were divided into three zones each, with the upper zone considered to be at and above the aortic arch, the middle zone between the aortic arch and inferior pulmonary vein, and the lower zone below the inferior pulmonary vein. The CT features included ground-glass opacity, centrilobular nodules, intralobular fine linear-reticular opacity and emphysema. Each of the patterns was scored on a scale of $0-4$, which represented zonal involvement of $0,1-25,26-50,5-75$ and $>75 \%$, respectively. The average score for HRCT abnormalities in each zone was calculated by averaging the score of each section in each zone, and then by averaging the mean score for the right and that left lungs. The overall score for HRCT abnormalities was first calculated by averaging the score of each section of the right and left lungs, and then by averaging the mean scores for the right and left lungs.

\section{Statistical analysis}

A paired t-test was used to analyse the differences in overall scores for each HRCT abnormality, as well as to analyse the differences between the pulmonary function test results and scoring results of each HRCT abnormality before and after stopping smoking. Spearman rank correlation coefficients were used to determine the association between the initial scoring result of each HRCT abnormality and the initial results of
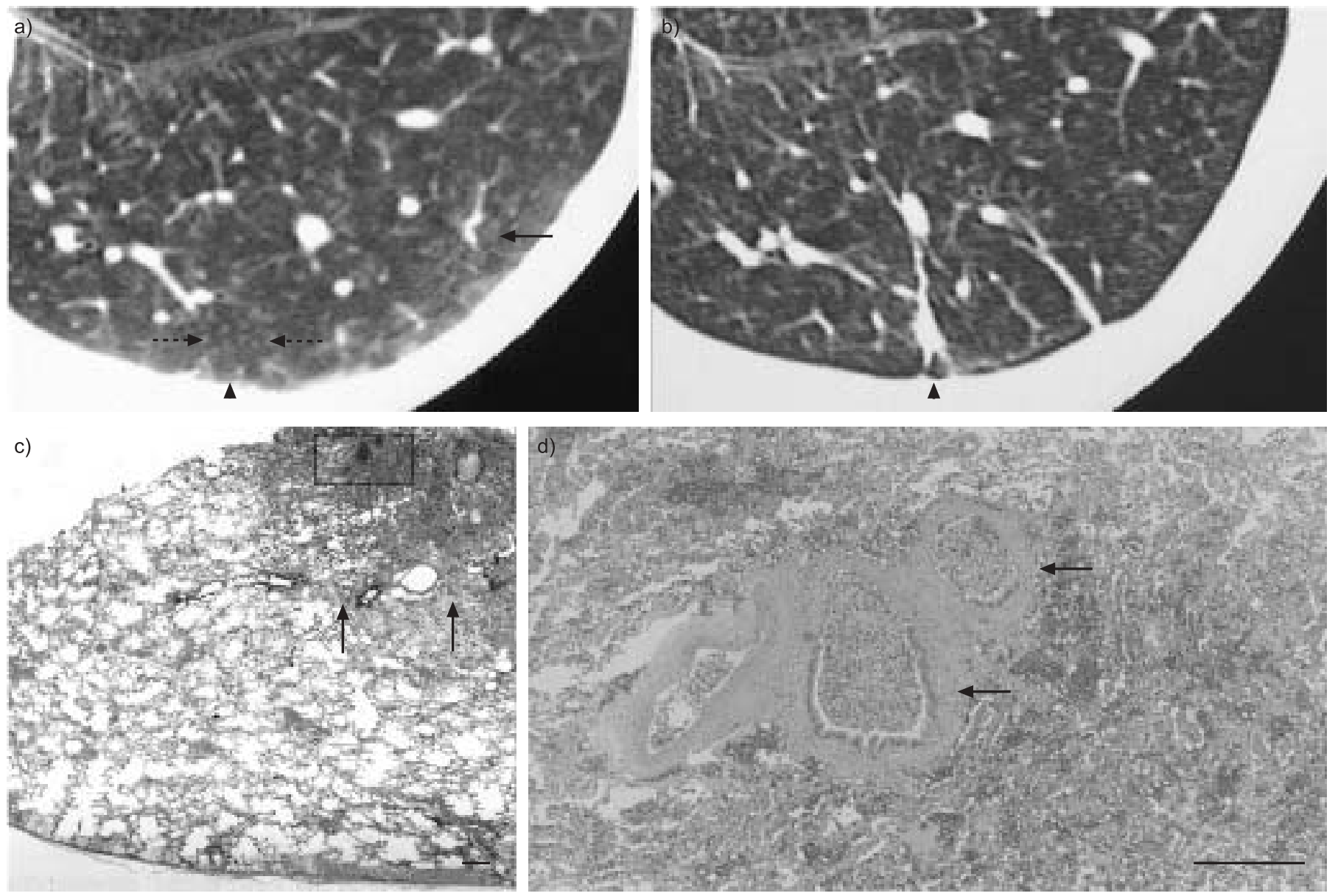

FIGURE 1. Changes in high-resolution computed tomography (HRCT) findings and pathological findings in a patient with respiratory bronchiolitis-associated interstitial lung disease (RB-ILD) in case 1. a) HRCT image taken at the time of biopsy, showing centrilobular micronodules (dashed arrows), centrilobular larger nodules (solid arrow) and ground-glass opacity. Centrilobular micronodules and centrilobular larger nodules are superimposed on areas of ground-glass opacity. The biopsy site is shown by an arrowhead. The pathological findings for this site are shown in c) and d). b) HRCT image taken 2 yrs after diagnosis showing significant improvements in ground-glass opacity, and centrilobular nodules and their superimposition. Scarring from the surgical lung biopsy is shown by arrowhead. c) Image of specimen sampled from lesion shown by arrowhead in a). Mild peribronchiolar inflammation and fibrosis in centrilobular lesions are shown (arrows). The enlarged figure in the quadrilateral section is shown in d) (haematoxylin and eosin staining; scale bar $=400 \mu \mathrm{m}$ ). d) Mucin and macrophages are shown packed in the bronchiole (arrows). Adjacent alveoli containing pigmented alveolar macrophages, along with mild peribronchiolar inflammation and fibrosis, are also shown (haematoxylin and eosin staining; scale bar $=400 \mu \mathrm{m}$ ). 

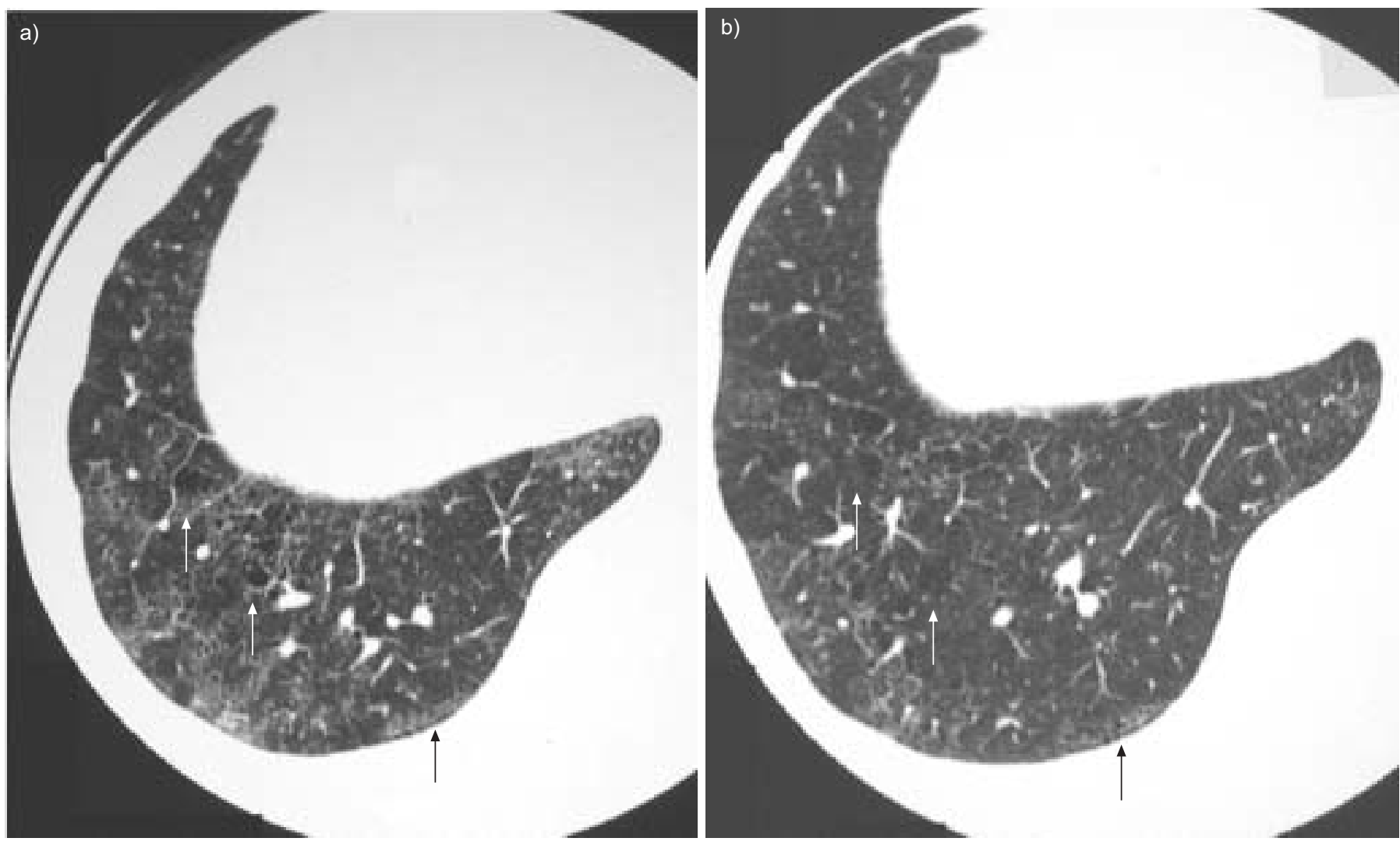

FIGURE 2. Changes in high-resolution computed tomography (HRCT) findings in a patient with respiratory bronchiolitis-associated interstitial lung disease (case 3). a) HRCT image taken at the time of biopsy showing ground-glass opacity, and centrilobular nodules and their superimposition. In addition, areas of subpleural intralobular fine reticular opacity (black arrow) and emphysema (white arrows) are noted. b) HRCT image taken 2 yrs after diagnosis showing significant improvement in ground-glass opacity, and centrilobular nodules and their superimposition. However, the findings remained in part, and the areas of subpleural intralobular fine reticular opacity (black arrow) and emphysema did not change. Emphysema became difficult to recognise by HRCT because the areas of ground-glass opacity, which can provide background density and accentuate such abnormalities, were decreased (white arrows).

pulmonary function testing, as well as to determine the associations between the changes in each pulmonary function test result and the changes in each HRCT scoring result following smoking cessation.

\section{RESULTS}

\section{Initial HRCT findings}

The major findings for the five RB-ILD cases, which were remarkable in each patient, were as follows: 1) centrilobular micronodules $<3 \mathrm{~mm}$ that were mostly well defined and of high density; 2) larger nodules that were mainly ill defined and hazy in appearance (ground-glass attenuation), with centrilobular distribution; and 3) areas of ground-glass attenuation (fig. 1a). The superimposition of centrilobular nodules on ground-glass opacity was notable in each case. In addition, minor findings included the following: 1) bronchial wall thickening $(n=5) ; 2)$ emphysema $(n=3$; fig. $2 a) ; 3)$ intralobular fine linear-reticular opacity $(n=3$; figs $2 a$ and $3 a) ; 4)$ areas of hypoattenuation $(n=3)$; and 5) traction bronchiolectasis $(n=1)$. Bronchial wall thickening was observed in all cases, although it was not prominent. Emphysema, intralobular fine linearreticular opacity and areas of hypoattenuation findings were partial, if observed. No expiratory HRCT examinations were performed with the patients.

\section{CT pathological correlations}

Centrilobular micronodules $(<3 \mathrm{~mm})$ on HRCT images (figs 1a and 3a) corresponded to findings of mucin and accumulation of macrophages packed in bronchioles (fig. 1c and $\mathrm{d}$ ), or bronchiolar fibrosis (fig. $3 \mathrm{c}$ and $\mathrm{d}$ ). Larger nodules $(>3 \mathrm{~mm})$ on HRCT images corresponded to peribronchial inflammation/fibrosis with an accumulation of macrophages within the alveolar air space. These lesions often accompanied scant alveolar inflammation in a neighbouring area or accumulation of macrophages within the alveolar air space (figs 1c and 3c), and accounted well for nodules that had an illdefined or hazy appearance.

Histological sections, taken from affected lesions with groundglass opacity, showed areas of macrophage accumulation within the alveolar air space and mild alveolar fibrosis/ inflammation. These pathological lesions in the RB-ILD patients had a patchy and centrilobular distribution (figs 1c and $3 c$ ), but were not diffuse and were likely to indicate a slightly increased attenuation, as depicted by HRCT (figs 1a and 3a). In addition, mucin and an accumulation of macrophages packed in the bronchioles or centrilobular fibrosis were seen within the lesions with patchy distribution, which accounted for the superimposition of centrilobular nodules on the areas of ground-glass opacity revealed by HRCT. 

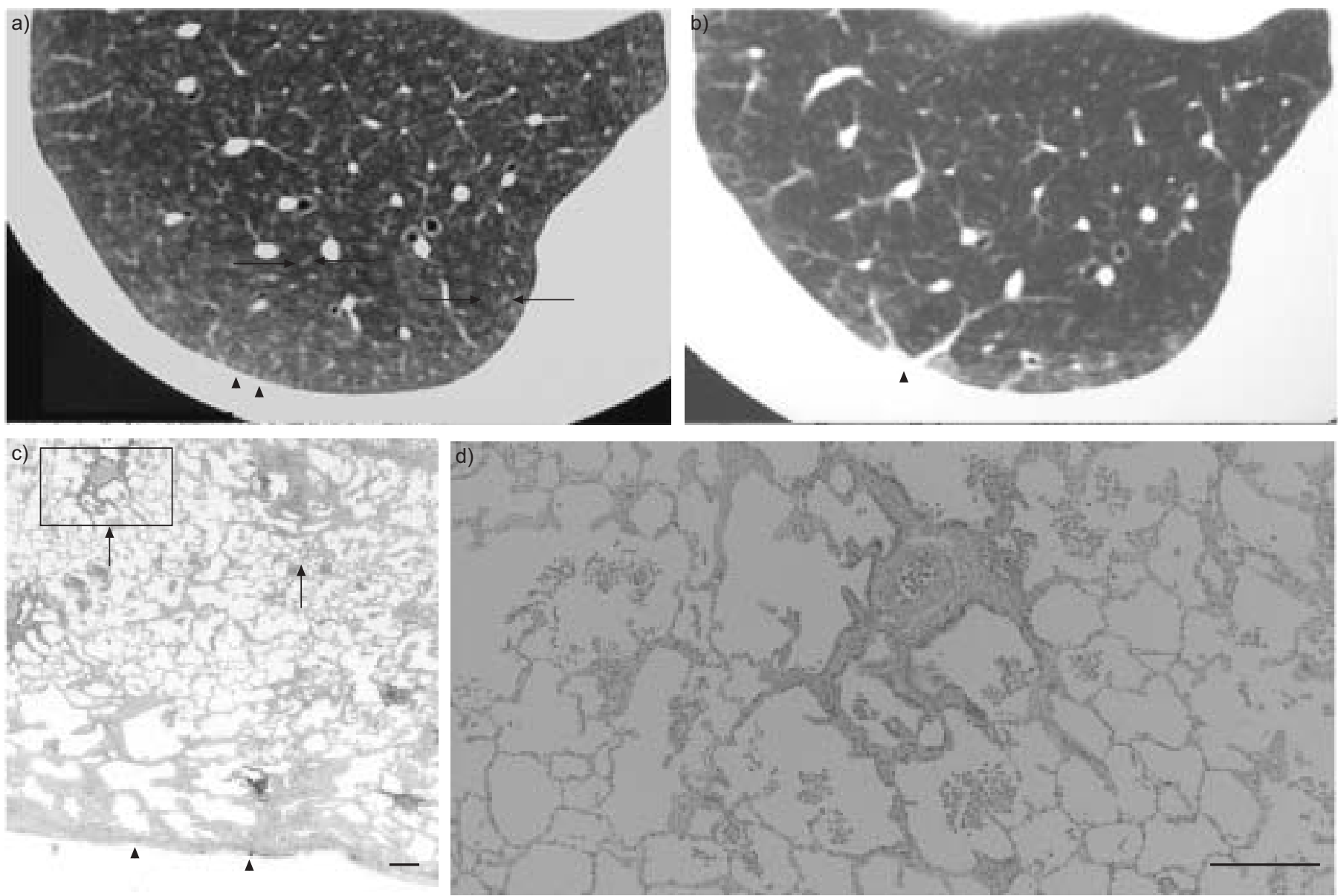

FIGURE 3. Changes in high-resolution computed tomography (HRCT) findings and pathological findings in a patient with respiratory bronchiolitis-associated interstitial lung disease (case 2). a) HRCT image taken at the time of biopsy showing ground-glass opacity, and centrilobular micronodules (arrows) and their superimposition. In addition, intralobular fine linear opacity can be seen in the subpleural lesions (arrowheads). This lesion was sampled by the surgical lung biopsy and pathological findings from the biopsy site are shown in c) and d). b) HRCT image taken 5 yrs after diagnosis showing significant improvement in ground-glass opacity, and centrilobular nodules and their superimposition. Scarring from the surgical lung biopsy is shown by arrowheads. Areas of intraloblar fine linear opacity initially observed were removed during the surgical lung biopsy procedure. c) The sampling site of this specimen is shown by the arrowheads in a). Mild peribronchiolar inflammation and fibrosis in centrilobular lesions are shown (arrows). Subpleural microcystic fibrotic changes are also noted (arrowheads). The enlarged figure in the quadrilateral section is shown in d (haematoxylin and eosin staining; scale bar $=400 \mu \mathrm{m})$. d) Bronchiole and adjacent alveoli containing pigmented alveolar macrophages along with peribronchiolar inflammation and fibrosis are shown (haematoxylin and eosin staining; scale bars $=400 \mu \mathrm{m}$ ).

Furthermore, intralobular linear-reticular opacity shown by the HRCT (fig. 3a) corresponded to fibrosis that involved the alveolar septa, including subpleural microcystic fibrosis (fig. 3c).

\section{Initial HRCT scores}

HRCT scores are given in table 2. The overall score for the centrilobular nodules was significantly higher than that of any other. The ground-glass opacity overall score was significantly higher than that of intralobular linear-reticular opacity and emphysema. With ground-glass opacity, centrilobular nodules and intralobular linear-reticular opacity, scores were highest in the lower zone and lowest in the upper. Conversely, the emphysema score was highest in the upper zone and lowest in the lower. The overall scores from the initial HRCT examinations were not statistically correlated with any of the initial pulmonary function test results (data not shown).

\section{Clinical course and changes in respiratory function test results}

Most symptoms in the patients disappeared gradually after smoking cessation. In the follow-up respiratory function tests, $D \mathrm{~L}, \mathrm{CO}$ alone was significantly improved. Arterial oxygen pressure also improved significantly, though it was slightly decreased initially (table 3).

\section{Changes in HRCT findings following smoking cessation}

Ground-glass opacity and centrilobular nodules were improved significantly in all cases (figs 1-3); however, the abnormalities partially remained and were varied in each individual. The intralobular fine linear-reticular patterns remained unchanged (fig. 2). Emphysema and traction bronchiolectasis also remained unchanged, though they became difficult to recognise on the HRCT images because the areas of ground-glass opacity, which provided background density and accentuated those abnormalities, were decreased (fig. 2). 


\begin{tabular}{lcccc} 
TABLE 2 & $\begin{array}{l}\text { Scores for initial high-resolution computed tomography abnormalities in five respiratory bronchiolitis-associated } \\
\text { interstitial lung disease patients }\end{array}$ \\
& Ground-glass opacity & Centrilobular nodules & Intralobular linear-reticular opacity & Emphysema \\
\hline Upper zone & $1.09 \pm 0.23$ & $1.78 \pm 0.61$ & $0.00 \pm 0.00$ & $1.04 \pm 0.82$ \\
Middle zone & $1.37 \pm 0.25$ & $2.42 \pm 0.44$ & $0.17 \pm 0.28$ & $0.54 \pm 0.42$ \\
Lower zone & $1.76 \pm 0.53$ & $2.58 \pm 0.57$ & $0.65 \pm 0.51$ & $0.38 \pm 0.48$ \\
Overall & $1.42 \pm 0.25^{\star}$ & $2.29 \pm 0.26^{\star \star}$ & $0.27 \pm 0.24$ & $0.54 \pm 0.43$ \\
\hline
\end{tabular}

Data are presented as abnormality scores for each zone and overall (mean $\pm \mathrm{SD}) .{ }^{*}$ : the overall score for ground-glass opacity was significantly higher than that of intralobular linear-reticular opacity and emphysema $(p<0.05)$; ${ }^{*}$ : the overall score for the centrilobular nodules was significantly higher than that of any other $(p<0.01)$

Furthermore, bronchial wall thickening and areas of hypoattenuation did not change significantly following smoking cessation. As for the scoring results, scores for the centrilobular nodules and ground-glass opacity were decreased significantly, while those for linear-reticular opacity and emphysema were not changed (table 3).

\section{Correlation between changes in HRCT scores and pulmonary function test results following smoking cessation}

The correlation between changes in HRCT scores and pulmonary function test results following smoking cessation are given in table 4 . The change in score for the centrilobular nodules was significantly negatively correlated with the change in DL,CO ( $\mathrm{p}=0.03$; fig. $4 \mathrm{a})$, while the change in score for ground-glass opacity tended to be negatively correlated with the change in $D \mathrm{~L}, \mathrm{CO}(\mathrm{p}=0.06)$. However, the change in score for the centrilobular nodules was not correlated with the change in arterial oxygen tension $\left(\mathrm{Pa}_{\mathrm{a}} \mathrm{O}_{2}\right.$; fig. $\left.4 \mathrm{~b}\right)$ and the change in $D \mathrm{~L}, \mathrm{CO}$ was not correlated with the change in $\mathrm{Pa}_{\mathrm{a}} \mathrm{O}_{2}$ (data not shown). In addition, there were no correlations between baseline $D \mathrm{~L}, \mathrm{CO} \%$ predicted and change in $\mathrm{DL}, \mathrm{CO} \%$ pred or between baseline $\mathrm{Pa}_{1} \mathrm{O}_{2}$ and change in $\mathrm{Pa}_{1} \mathrm{O}_{2}$ (fig. $4 \mathrm{c}$ and $\mathrm{d}$ ).

\section{DISCUSSION}

The HRCT findings and CT-pathological correlations in the present study are similar to those reported previously [9, 13, $15,17,21]$. All five of the present study's patients with RB-ILD showed clear improvements of abnormalities on HRCT images, as well as clinical symptoms and results of pulmonary function tests, following smoking cessation without corticosteroid therapy. The present results indicate that the most fundamental HRCT findings for RB-ILD are centrilobular nodules, ground-glass attenuation and superimposition of these two findings, which correspond to the pathological findings of RB. Furthermore, pathological findings associated with RB can be improved by smoking cessation, and such improvement leads to improvements in clinical symptoms, HRCT abnormalities and pulmonary function test results. In a longitudinal follow-up study of patients with smoker's lung [22], ground-glass attenuation and ill-defined micronodules on HRCT images were reduced in some of those who had stopped smoking. That reduction of abnormalities in patients with subclinical RB was considered to be essentially the same as in the present study's cases of clinical RB-ILD. However, it is difficult to objectively distinguish between RB and RB-ILD using HRCT or pathological findings alone, without an evaluation of clinical symptoms or respiratory function tests [23].

Visual and semiquantitative methods of scoring HRCT abnormalities are useful to investigate the relationships between HRCT abnormalities and the respiratory function test results, as well as to evaluate the progression of disease and response to treatment [24]. The results of respiratory function tests in RB-ILD patients vary in published reports [9, 12, 15]. In the present study's patients, there was no significant relationship

\begin{tabular}{|c|c|c|c|}
\hline & Before smoking cessation & After smoking cessation & p-value \\
\hline VC \% pred & $112.5 \pm 11.8$ & $109.4 \pm 9.0$ & 0.22 \\
\hline FEV1/FVC \% & $86.8 \pm 11.6$ & $91.6 \pm 6.1$ & 0.23 \\
\hline DL,Co \% pred & $52.2 \pm 16.9$ & $75.9 \pm 18.4$ & 0.03 \\
\hline $\mathrm{Pa}, \mathrm{O}_{2} \mathrm{mmHg}$ & $79.8 \pm 4.0$ & $86.0 \pm 3.5$ & 0.02 \\
\hline Score for ground-glass opacity & $1.42 \pm 0.25$ & $0.59 \pm 0.19$ & 0.03 \\
\hline Score for centrilobular nodules & $2.29 \pm 0.26$ & $0.67 \pm 0.15$ & 0.004 \\
\hline Score for linear-reticular opacity & $0.28 \pm 0.24$ & $0.27 \pm 0.24$ & 0.62 \\
\hline Score for emphysema & $0.55 \pm 0.43$ & $0.59 \pm 0.42$ & 0.43 \\
\hline
\end{tabular}

VC: vital capacity; \% pred: \% predicted; FEV1: forced expiratory volume in one second; FVC: forced vital capacity; DL,Co: diffusing capacity of the lung for carbon monoxide; $\mathrm{Pa}, \mathrm{O}_{2}$ : arterial oxygen tension $1 \mathrm{mmHg}=0.133 \mathrm{kPa}$ 


\begin{tabular}{|c|c|c|c|c|c|}
\hline \multirow[t]{2}{*}{ TABLE 4} & \multicolumn{5}{|c|}{$\begin{array}{l}\text { Correlations between changes in high-resolution computed tomography (HRCT) scores and pulmonary function test } \\
\text { results }\end{array}$} \\
\hline & & Change in $D L, C O$ & Change in VC \% pred & Change in FEV $1 / F V C$ & Change in $\mathrm{Pa}, \mathrm{O}_{2}$ \\
\hline \multicolumn{2}{|c|}{$\begin{array}{l}\text { Change in HRCT score for ground-glass } \\
\text { opacity }\end{array}$} & $-0.88(0.06)$ & $0.80(0.11)$ & $-0.45(0.48)$ & $-0.49(0.44)$ \\
\hline \multicolumn{2}{|c|}{$\begin{array}{l}\text { Change in HRCT score for centrilobular } \\
\text { nodules }\end{array}$} & $-0.91(0.03)$ & $0.54(0.38)$ & $-0.17(0.81)$ & $-0.59(0.34)$ \\
\hline \multicolumn{2}{|c|}{$\begin{array}{l}\text { Changes in HRCT score for intralobular } \\
\text { linear-reticular opacity }\end{array}$} & $-0.59(0.33)$ & $0.73(0.16)$ & $-0.57(0.36)$ & $-0.05(0.94)$ \\
\hline \multicolumn{2}{|c|}{ Changes in HRCT score for emphysema } & $0.28(0.68)$ & $-0.39(0.55)$ & $0.38(0.57)$ & $0.64(0.28)$ \\
\hline
\end{tabular}

between the score of initial abnormalities on HRCT and results of initial pulmonary function tests. It is likely that RB-ILD patients have some degree of emphysema or pulmonary fibrosis, thus the results of pulmonary function tests vary depending on those components. However, DL,CO in respiratory function tests and scores for the centrilobular nodules and ground-glass opacity on HRCT were improved significantly following smoking cessation in all of the present RB-ILD patients. Furthermore, the change in DL,CO was significantly negatively correlated with the change in score for centrilobular nodules on HRCT and tended to be negatively correlated with that of ground-glass opacity on HRCT. Therefore, the present authors believe that the improvement in $D \mathrm{~L}, \mathrm{CO}$ in the present patients reflected improvement of the centrilobular nodules and ground-glass opacity on HRCT following smoking cessation. Nevertheless, this issue should be clarified by investigating a greater number of subjects.

In contrast, the change in $\mathrm{Pa}_{1} \mathrm{O}_{2}$ was not significantly correlated with the change in score of centrilobular nodules on HRCT, though that was improved significantly after smoking cessation. The decrease in $\mathrm{Pa}_{1} \mathrm{O}_{2}$ may not only have been due to the decrease in $\mathrm{DL}, \mathrm{CO}$ but also because of the involvement of an airway obstruction or inflammation, though the values for obstructive disturbance in all five patients were minimal. The present authors consider that if the study were repeated with a larger population, a correlation would be seen between changes in $\mathrm{Pa}, \mathrm{O}_{2}$ and the same HRCT score. In addition, the improvement in the forced expiratory volume in one second/forced vital capacity ratio would become significant. An additional study with a larger cohort is needed to clarify this issue.

RB-ILD is difficult to distinguish from DIP [8-13]. In the present study's patients, inflammatory and mild fibrotic lesions were limited in the centrilobular lesions, with patchy distribution observed, although subpleural mild fibrotic cystic changes were occasionally associated. Pathological features of DIP, such as diffuse alveolar wall thickening and accumulation of macrophages in the alveolar space, were not noted in any of the biopsy specimens from the present five patients. In addition, the HRCT findings were considered to be different from those of DIP because HRCT features of DIP include mainly ground-glass opacity and scarce centrilobular nodules
$[13,25]$. The present HRCT findings resembled those of subacute hypersensitivity pneumonitis or flock workers' lung rather than DIP, and the same characteristic HRCT features have been reported in previous articles [26, 27]. Furthermore, the BAL fluid findings in the present patients were different from those of DIP, which often reveal an increased number of lymphocytes or neutrophils [23, 28].

Originally, RB was postulated to be a precursor of centriacinar emphysema [1]. In a study of serial HRCT findings in smokers [23], it was reported that profuse micronodules on HRCT images had been replaced by emphysema in some of the subjects, although WELLS et al. [29] noted that those observations should require reproduction with a larger patient cohort. In the present cases of smoking cessation, no replacement of centrilobular nodules with emphysema was observed. In the initial HRCT images of the present patients, the scores for centrilobular nodules, ground-glass opacity and intralobular linear-reticular opacity were increased in the order of upper, middle and lower zone, whereas the scores for emphysema were decreased in that order. Thus, RB might develop into pulmonary fibrosis rather than emphysema; however, a study with a larger number of cases is needed to clarify this notion.

It is known that some cases of RB-ILD show an unchanged or deteriorated condition after smoking cessation and corticosteroid therapy $[9,12]$. RB has been found in subjects many years after stopping smoking [11]. In the present study, lesions partly remained after smoking cessation, with some variation among the individual patients. The degree of improvement shown by HRCT may depend on the period of time following smoking cessation and intensity of inflammation/fibrosis. A previous study noted that when findings in patients diagnosed with RBILD are not compatible with RB on HRCT images or in biopsy specimens they may be determinant and those of RB may only be incidental [9]. RB-ILD patients with more advanced lung fibrosis may not improve significantly through smoking cessation alone. Although all the present patients had a small amount of emphysema or lung fibrosis, the present authors believe that they showed characteristic features of RB-ILD.

Although the present authors have occasionally treated patients who show clinical and radiological findings similar to those in the present study, they do not usually recommend a 

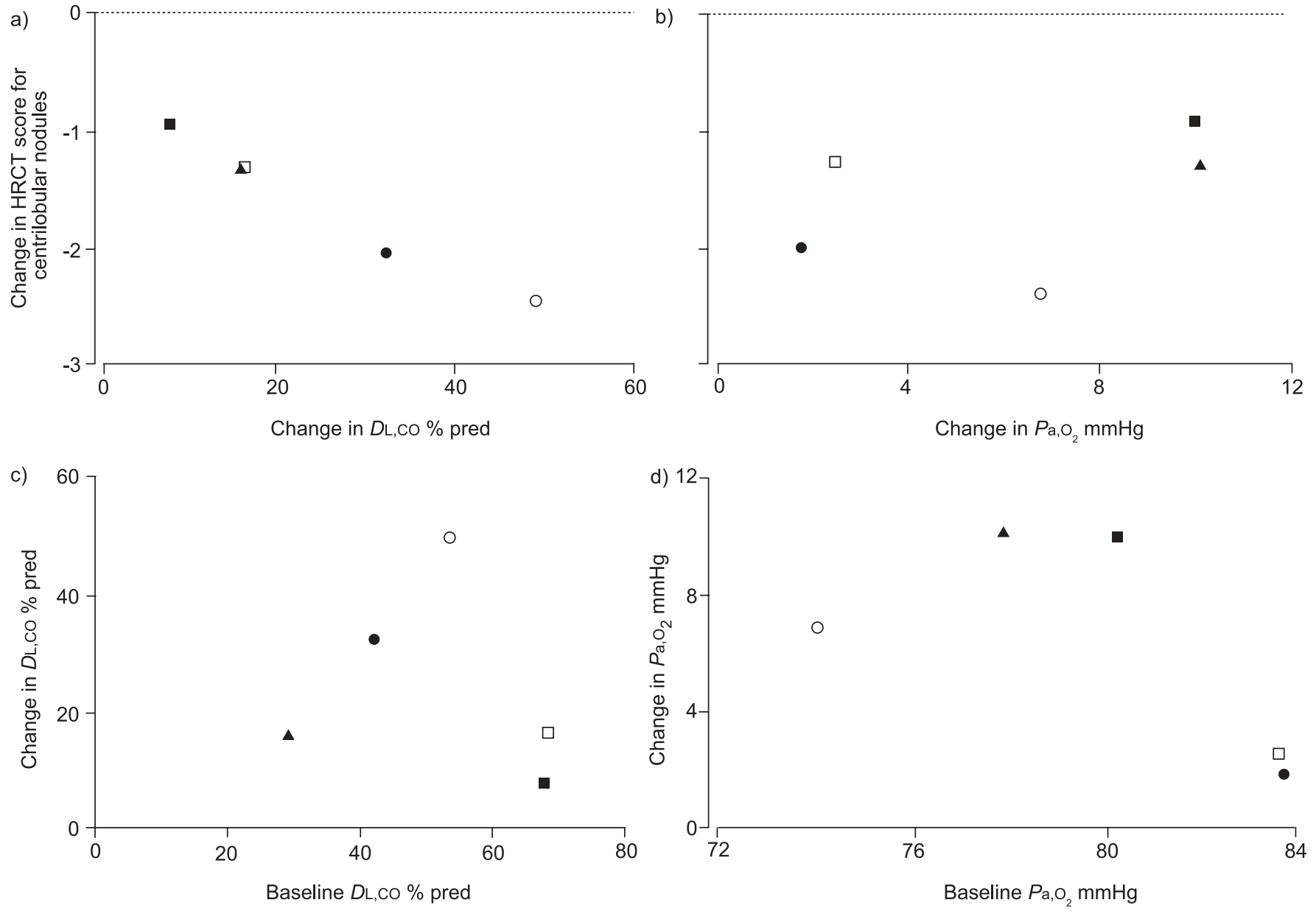

FIGURE 4. a) Relationship between changes in the diffusing capacity of the lung for carbon monoxide $(D L, C O) \%$ predicted and high-resolution computed tomography (HRCT) score for centrilobular nodules. b) Relationship between changes in arterial oxygen tension $\left(\mathrm{Pa}_{\mathrm{a}} \mathrm{O}_{2}\right)$ and $\mathrm{HRCT}$ score for centrilobular nodules. c) Relationship between baseline $D \mathrm{~L}, \mathrm{CO} \%$ pred and change in $D \mathrm{~L}, \mathrm{CO} \%$ pred. d) Relationship between baseline $\mathrm{Pa}_{\mathrm{a}} \mathrm{O}_{2}$ and change in $P_{\mathrm{a}, \mathrm{O}_{2}}$. $\square$ : case 1 ; $\boldsymbol{\bullet}$ : case $2 ; \mathbf{\Lambda}$ : case 3; $\bigcirc$ : case 4 ; $\mathbf{\square}$ : case 5 .

surgical lung biopsy because the symptoms in most are not severe. The present patients agreed with the authors' recommendation of a surgical lung biopsy for diagnosis and stopped smoking after being informed of the results. The present authors believe that they were able to confirm smoking cessation by interviewing the patients and their family members, as well as by the improvements seen, though cotinine assays were not performed.

In summary, the present study followed five respiratory bronchiolitis-associated interstitial lung disease patients for a range of $15-62$ months $(46.4 \pm 20.8$ months $)$ after smoking cessation. Clinical symptoms, respiratory functional abnormalities and high-resolution computed tomography abnormalities in those patients were found to be improved by smoking cessation alone, and it was concluded that ground-glass opacity and centrilobular nodules, as shown by high-resolution computed tomography, representing pathological respiratory bronchiolitis, can be improved by stopping a smoking habit. However, findings of fibrosis, which may represent other forms of interstitial lung disease, were not improved. It remains unclear whether respiratory bronchiolitis advances to emphysema or other forms of interstitial lung diseases and whether there is another pathological factor of respiratory bronchiolitisassociated interstitial lung disease in addition to smoking.

\section{REFERENCES}

1 Niewoehner DE, Kleinerman J, Rice DB, Div M. Pathologic changes in the peripheral airway of young cigarette smokers. N Engl J Med 1974; 291: 755-758.

2 Colby TV. Bronchiolitis: pathologic considerations. Am J Clin Pathol 1998; 109: 101-109.

3 Cottin V, Streichenberger N, Gamondes J-P, Thevenet F, Loire R, Cordier JF. Respiratory bronchiolitis in smokers with spontaneous pneumothorax. Eur Respir J 1998; 12: 702-704.

4 Remy-Jardin M, Remy J, Boulenguez C, Sobaszek A, Edme J-L, Furon D. Morphologic effects of cigarette smoking on airways and pulmonary parenchyma in healthy adult volunteers: CT evaluation and correlation with pulmonary function tests. Radiology 1993; 186: 107-115.

5 Myers JL, Veal CF Jr, Shin MS, Katzenstein ALA. Respiratory bronchiolitis causing interstitial lung disease: a clinicopathologic study of six cases. Am Rev Respir Dis 1987; 135: 880-884. 
6 Yousem SA, Colby TV, Gaensler EA. Respiratory bronchiolitis-associated interstitial lung disease and its relationship to desquamative interstitial pneumonia. Mayo Clin Proc 1989; 64: 1373-1380.

7 American Thoracic Society/European Respiratory Society International Multidisciplinary Consensus Classification of the idiopathic interstitial pneumonias. Am J Respir Crit Care Med 2002; 165: 277-304.

8 Katzenstein ALA, Myers JL. Idiopathic pulmonary fibrosis: clinical relevance of pathologic classification. Am J Respir Crit Care Med 1998; 157: 1301-1315.

9 Moon J, du Bois RM, Colby TV, Hansell DM, Nicholson AG. Clinical significance of respiratory bronchiolitis on open lung biopsy and its relationship to smoking related interstitial lung disease. Thorax 1999; 54: 1009-1014.

10 Craig PJ, Wells AU, Doffman S, et al. Desquamative interstitial pneumonia, respiratory bronchiolitis and their relationship to smoking. Histopathology 2004; 45: 275-282.

11 Fraig M, Shreesha U, Savici DR, Katzenstein ALA. Respiratory broncholitis. Am J Surg Pathol 2002; 26: 647653.

12 Ryu JH, Myers JL, Capizzi SA, Douglas WW, Vassallo R, Decker PA. Desquamative interstitial pneumonia and respiratory bronchiolitis-associated interstitial lung disease. Chest 2005; 127: 178-184.

13 Heyneman LE, Ward S, Lynch DA, Remy-Jardin M, Johkoh T, Muller NL. Respiratory bronchiolitis, respiratory bronchiolitis-associated interstitial lung disease, and desquamative interstitial pneumonia: different entities or part of the spectrum of the same disease process? AJR 1999; 173: 1617-1622.

14 King TE. Respiratory bronchiolitis-associated interstitial lung disease. Clin Chest Med 1993; 14: 693-698.

15 Park JS, Brown KK, Tuder RM, Hale VAE, King TE, Lynch DA. Respiratory bronchiolitis-associated interstitial lung disease: radiologic features with clinical and pathologic correlation. J Comput Assist Tomogr 2002; 26: 13-20.

16 Sadikot RT, Johnson J, Loyd JE, Christman JW. Respiratory bronchiolitis associated with severe dyspnea, exertional hypoxemia, and clubbing. Chest 2000; 117: 282-285.

17 Holt RM, Schmidt RA, Godwin JD, Raghu G. High resolution $\mathrm{CT}$ in respiratory bronchiolitis-associated interstitial lung disease. J Comput Assist Tomogr 1993; 17: 46-50.
18 American Thoracic Society/European Respiratory Society. Idiopathic pulmonary fibrosis: diagnosis and treatment; international consensus statement. Am J Respir Crit Care Med 2000; 161: 646-664.

19 Hansell DM, Wells AU, Padley SP, Muller NL. Hypersensitivity pneumonitis: correlation of individual CT patterns with functional abnormalities. Radiology 1996; 199: 123-128.

20 Kazerooni EA, Martinez FJ, Flint A, et al. Thin-section CT obtained at $10-\mathrm{mm}$ increments versus limited three-level thin-section CT for idiopathic pulmonary fibrosis: correlation with pathologic scoring. AJR 1997; 169: 977-983.

21 Remy-Jardin M, Remy J, Gosselin B, Becette V, Edme JL. Lung parenchymal changes secondary to cigarette smoking: pathologic-CT correlations. Radiology 1993; 186: 643651.

22 Remy-Jardin M, Edme J-L, Boulenguez C, Remy J, Mastora I, Sobaszek A. Longitudinal follow-up study of smoker's lung with thin-section $\mathrm{CT}$ in correlation with pulmonary function tests. Radiology 2002; 222: 261-270.

23 Davies G, Wells AU, du Bois RM. Respiratory bronchiolitis associated with interstitial lung disease and desquamative interstitial pneumonia. Clin Chest Med 2004; 25: 717-726.

24 Lynch DA, Newell JD Jr. Quantification of diffuse lung diseases. In: Lynch DA, Newell JD Jr, Lee JS, eds. Imaging of Diffuse Lung Disease. London, B.C. Decker Inc, 2000; pp. 303-314.

25 Hartman TE, Primack SL, Swensen SJ, Hansell D, McGuinness G, Muller NL. Desquamative interstitial pneumonia: thin-section CT findings in 22 patients. Radiology 1993; 187: 787-790.

26 Desai SR, Ryan SM, Colby TV. Smoking-related interstitial lung disease: histopathological and imaging perspectives. Clin Radiol 2003; 58: 259-268.

27 Weiland DA, Lynch DA, Jensen SP, et al. Thin-section CT findings in flock worker's lung, a work-related interstitial lung disease. Radiology 2003; 227: 222-231.

28 Elkin SL, Nicholson AG, Path MRC, du Bois RM. Desquamative interstitial pneumonia and respiratory bronchiolitis-associated interstitial lung disease. Semin Respir Crit Care Med 2001; 22: 387-397.

29 Wells AU, Nicholson AG, Hansell DM, du Bois RM. Respiratory bronchiolitis-associated interstitial lung disease. Semin Respir Crit Care Med 2003; 24: 585-594. 